\title{
Advances in modeling polarimeter performance
}

\section{Russell A. Chipman}

Russell A. Chipman, "Advances in modeling polarimeter performance," Proc. SPIE 10407, Polarization Science and Remote Sensing VIII, 1040707 (30 August 2017); doi: 10.1117/12.2274506

EDent: SPIE Optical Engineering + Applications, 2017, San Diego, California, United States 


\title{
Advances in Modeling Polarimeter Performance
}

\author{
Russell A. Chipman \\ University of Arizona, College of Optical Sciences, 1630 E. University Drive, Tucson, AZ, USA \\ 85718
}

\begin{abstract}
Artifacts in polarimeters are apparent polarization features which are not real but result from the systematic errors in the polarimeter. The polarization artifacts are different between division of focal plane, spectral, and time modulation polarimeters. Artifacts result from many sources such as source properties, micropolarizer arrays, coatings issues, vibrations, and stress birefringence. A modeling examples of polarization artifacts due to a micro-polarizer array polarimeter is presented.
\end{abstract}

Keywords: Polarization, coronagraph, imaging, polarization aberration, polarization ray tracing

\section{INTRODUCTION}

Polarimeters are optical instruments for measuring the polarization properties of light beams and samples. Polarimetry, the science of polarization measurement, is most simply characterized as radiometry with polarization elements. Accurate polarimetry requires careful attention to all the issues necessary for accurate radiometry, together with many additional polarization issues which must be mastered to accurately determine polarization properties from polarimetric measurements. Light-measuring polarimeters measure the polarization state of a beam of light and its polarization characteristics: the Stokes parameters, the direction of oscillation of the electric field vector for a linearly polarized beam, the helicity of a circularly polarized beam, the elliptical parameters of an elliptically polarized beam, and the degree of polarization. Sample-measuring polarimeters determine the relationship between the polarization states of incident upon and exiting a sample, and infer the polarization characteristics of the sample, its diattenuation, retardance, and depolarization, from the measurements. The term exiting beam is general and in different measurements might describe beams which are transmitted, reflected, diffracted, scattered or otherwise modified. The term sample is also an inclusive term used in a broad sense to describe a general light-matter interaction or sequence of such interactions and applies to practically anything.

Accurate polarimetric measurements can be made only if the polarization generator and polarization analyzer are well calibrated; the polarization states exiting the generator must be well known, as are the states analyzed by the analyzer. To perform accurate polarimetry, the polarization elements need not be ideal or of the highest quality. If the Mueller matrices of the polarization components are known from careful calibration, the systematic errors due to non-ideal polarization elements are removed during the data reduction.

\section{EQUATIONS OF POLARIMETRY}

The polarization analyzer consists of the polarization elements used for analyzing the polarization state, any other optical elements (lenses, mirrors, etc.) following the polarization elements, and the polarimeter's detector. The polarization effects from all elements are included in the measurement and data reduction procedures. A polarization analyzer is characterized by an analyzer vector containing four elements, defined analogously to the Stokes parameters. The polarization analyzer's analyzer vector $\mathbf{A}$ is

$$
\mathbf{A}=\left(\begin{array}{l}
a_{0} \\
a_{1} \\
a_{2} \\
a_{3}
\end{array}\right)=\left(\begin{array}{c}
P_{H}+P_{V} \\
P_{H}-P_{V} \\
P_{45}-P_{135} \\
P_{R}-P_{L}
\end{array}\right)
$$

Polarization Science and Remote Sensing VIII, edited by Joseph A. Shaw, Frans Snik, Proc. of SPIE Vol. 10407, 1040707 · (c) 2017 SPIE · CCC code: 0277-786X/17/\$18 doi: $10.1117 / 12.2274506$ 
Note that in the absence of noise, $P_{H}+P_{V}=P_{45}+P_{135}=P_{R}+P_{L}$. The response $P$ of the polarization analyzer to an arbitrary polarization state $\mathbf{S}$ is a dot product of the analyzer vector with the incident Stokes parameters,

$$
P=\mathbf{A} \cdot \mathbf{S}=a_{0} S_{0}+a_{1} S_{1}+a_{2} S_{2}+a_{3} S_{3} .
$$

The analyzer vector will be the top row of the Mueller matrix for the polarization analyzer. A Stokes parameters measurement is a set of measurements acquired with a set of polarization analyzers placed into the beam of light. Let the total number of analyzers be $Q$, with each analyzer $\mathbf{A}_{q}$ specified by index $q=0,1, \ldots, Q-1$. Assume the incident Stokes parameters are the same for all measurements. The $q^{\text {th }}$ measurement generates an output, a flux measurement $P_{q}=\mathbf{A}_{q} \cdot \mathbf{S}$. A polarimetric measurement matrix $\mathbf{W}$ is defined as a $Q \times 4$ matrix with the $q^{\text {th }}$ row containing the analyzer vector $\mathbf{A}_{q}$,

$$
\mathbf{P}=\left(\begin{array}{c}
P_{0} \\
P_{1} \\
\vdots \\
P_{Q-1}
\end{array}\right)=\mathbf{W} \cdot \mathbf{S}=\left(\begin{array}{cccc}
a_{00} & a_{01} & a_{02} & a_{03} \\
a_{10} & a_{11} & a_{12} & a_{13} \\
\vdots & & & \\
a_{Q-1,0} & a_{Q-1,1} & a_{Q-1,2} & a_{Q-1,3}
\end{array}\right) \cdot\left(\begin{array}{c}
S_{0} \\
S_{1} \\
S_{2} \\
S_{3}
\end{array}\right) .
$$

To calculate the Stokes parameters from the data, the inverse of $\mathbf{W}$ is determined and applied to the measured data. The measured value for the incident Stokes parameters $\mathbf{S}_{m}$ is related to the data by the polarimetric data reduction matrix $\mathbf{W}^{-}$ 1,

$$
\mathbf{W}^{-1} \cdot \mathbf{P}=\mathbf{S}_{m}
$$

This is the polarimetric data reduction equation. During the setup of a Stokes polarimeter, the analyzer vectors are established. This calibration determines the rows of $\mathbf{W}$ and thus $\mathbf{W}^{-1}$. W' $\mathbf{W}^{-1}$ is then applied routinely to the measurements $\mathbf{P}$ to calculate the Stokes parameters.

\section{POLARIZATION ARTIFACTS IN IMAGING POLARIMETERS}

Artifacts in polarimeters are apparent polarization features which are not real but a result of the systematic errors in the polarimeter. A simple way to understand artifacts is to picture the polarimeter measuring a completely unpolarized scene. Any polarization present in the polarimetric image is thus due to some error in the polarimeter operation or data reduction. Polarization artifacts are frequently associated with motion in the scene, intensity gradients near edges of the scene, and drift of the polarimeter's analyzer vectors between calibration and operation. Artifacts are ubiquitous in polarimetric data. A thorough understanding can avoid erroneous interpretation of polarimetric data in cases where artifacts are expected.

Imaging polarimeters are particularly susceptible to misalignment of the raw images, since polarization properties are determined from the difference between flux measurements. Such misalignment causes polarization artifacts in the image on account of spurious polarization mixed with the actual polarization. Raw image misalignments occur due to source motion, polarimeter motion, vibration, and beam wander from slight wedge in rotating components. Polarization artifacts are largest in areas where the image intensity is changing the fastest, around object edges and near point sources. The edges of objects are usually where the angles of incidence and angles of scatter are larger. The largest polarization is typically expected around these areas. Due to vibration, image motion, and image misalignment, these are also the areas where the data is most suspect. Other errors result from imperfect polarization elements, and detector noise.

When the source flux fluctuates between raw images, a uniform polarization error occurs across the entire image. Source fluctuations are a serious problem in outdoor Stokes imagery because sunlight fluctuates due to cloud motion across the sun. Since imaging polarimeters need to acquire several images of a scene through different analyzers, there is an opportunity for error when the fields of view for a particular pixel do not coincide in position, time or wavelength.

Consider a rotating retarder imaging polarimeter taking sequential measurements in time. If the retarder has some wedge, non-parallel surfaces, it acts as a weak prism deflecting the light. As the retarder rotates between measurements, the image undergoes a small circle on the focal plane, and as a result, at each pixel the field of view wobbles slightly. If the object is of uniform intensity and polarization, this circling motion makes little difference. But when there is an 
intensity gradient, particularly around point sources and edges, the measured light flux will fluctuate, the data reduction will miscalculate, and generate a polarization artifact.[1 $]^{\mathrm{i}}$

The polarization artifacts are much larger with division of focal plane polarimeters since adjacent pixels have different analyzers, and several pixels are needed to perform each polarization measurement.[2] $]^{\text {ii }}$ Division of focal plane polarimetry uses arrays of polarization analyzers incorporated above a focal plane such that neighboring pixels analyze different polarization states. These are also known as micro-polarizer array polarimeters, since they incorporate arrays of pixel-sized micro-polarizers integrated with a focal plane array, such as a CCD or CMOS image sensor.[3],[4],[5] $]^{\mathrm{ii}, \mathrm{iv}, \mathrm{v}}$ An example micro-polarizer array configuration is shown in Figure 1 (left) with a repeating array of four linear polarizers oriented at $0^{\circ}, 45^{\circ}, 90^{\circ}$, and $135^{\circ}$ positioned above a focal plane. The earliest configurations used infrared wire grid polarizers $[6]^{\mathrm{vi}}$ which are easier to fabricate than visible wire grid polarizers due to the larger wire sizes and wire pitch. As wire grid polarizer technology advanced, visible wire grid polarizers and micro-polarizer arrays were developed.[7] ${ }^{\text {vii }}$ Many polarizer configurations can be used and the analyzers can be linear, elliptical, or circular. Flux measurements from several pixels are needed to calculate the Stokes parameters at a small group of pixels, then the data reduction is repeated across the image. An example imaging Mueller matrix measurement of a micro-polarizer array is shown in Figure 1 (right) where the $M_{01}$ and $M_{10}$ elements show the $0^{\circ}$ and $90^{\circ}$ polarizer elements and the $M_{02}$ and $M_{20}$ elements show the $45^{\circ}$ and $135^{\circ}$ polarizer elements. For this micro-polarizer array, the polarizer elements are all linear; no circular components measured and thus this is an incomplete polarimeter.
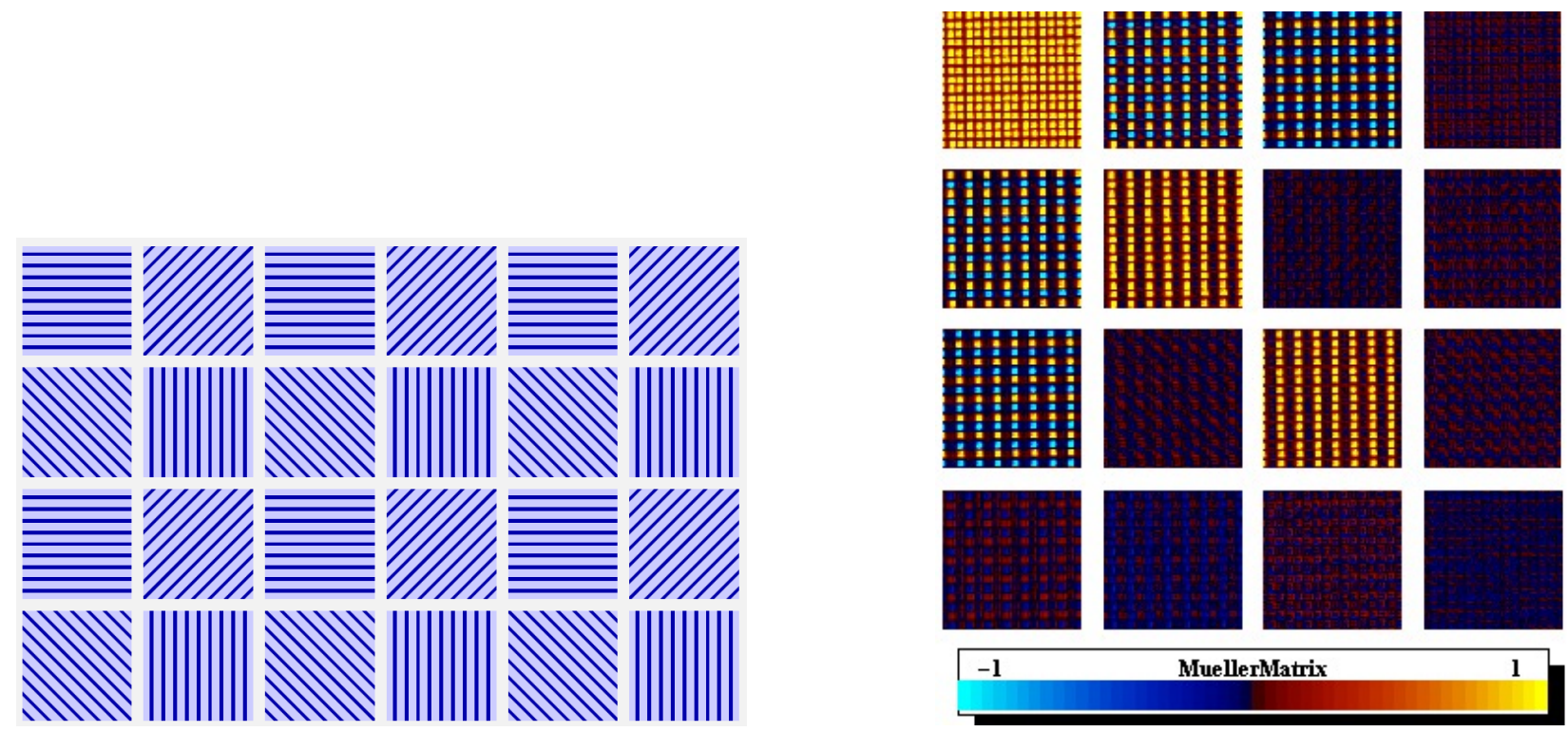

Figure 1. (Left) A micro-polarizer array has a repeating set of polarizers and is suitable to be placed over a focal plane array such that adjacent pixels analyze different polarization states. (Right) A Mueller matrix image of a micro-polarizer array. The $M_{00}$ image (upper left) shows the apertures of the polarizers as yellow and opaque regions as dark red. In the $M_{01}$ and $M_{10}$ images, the yellow squares are $0^{\circ}$ polarizers and the blue squares are $90^{\circ}$ polarizers. Similarly in the $M_{02}$ and $M_{20}$ images, the yellow squares are $45^{\circ}$ polarizers and the blue squares are $135^{\circ}$ polarizers. Ideally, the last column and the last row would be identically zero. Quantitative analysis of this image provides precise measurements of polarizer diattenuation and orientation errors.

As an example of micropolarizer array polarization artifacts, Figure 2 is a $660 \mathrm{~nm}$ image of a parking lot taken with the MSPI polarimeter. The corresponding DoLP is shown in Figure 3 (left). In Figure 3 (right) the DoLP data has been reduced to simulate a micro-polarizer array imaging polarimeter with a polarizer mask of the form of Figure 1 (left). All the differences between the right and left sides are polarization artifacts from the micropolarizer array. In the right $D o L P$ image artifacts occur around the edges of all the objects and shadows. Figure 4 compares the measured MSPI $S_{2}$ image to the corresponding $S_{2}$ image with the micro-polarizer array polarimeter. 


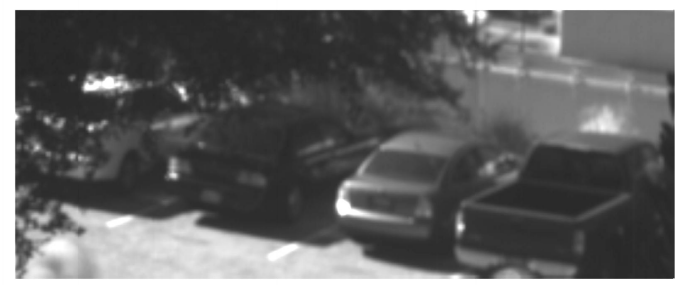

Figure 2. An irradiance image of cars in a parking lot.
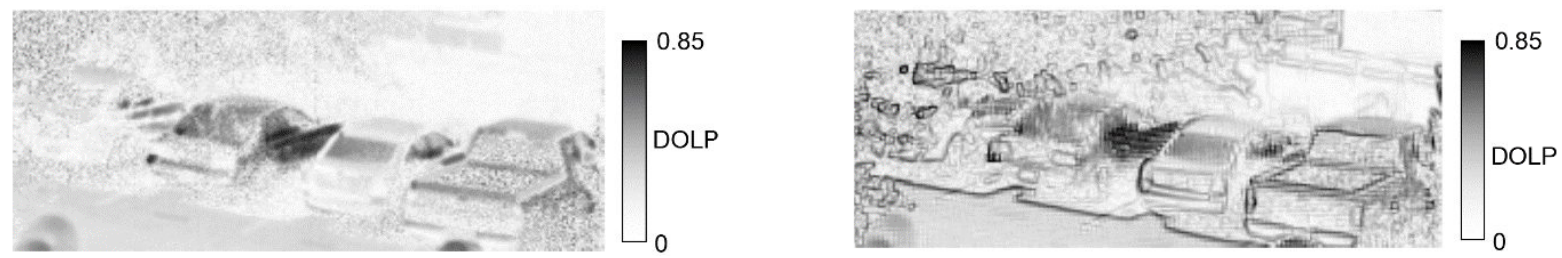

Figure 3. (Left) The DoLP image of Figure 2. White areas have low DoLP. (Right) The DoLP image as measured by a division of focal plane polarimeter. Any difference between the left and right images is a polarization artifact. The artifacts are significant around all bright edges, such as around the edges of the cars and the edges of their shadows, as well as artifacts in the tree. The division of focal plane polarization artifacts have a signature checkerboard pattern.
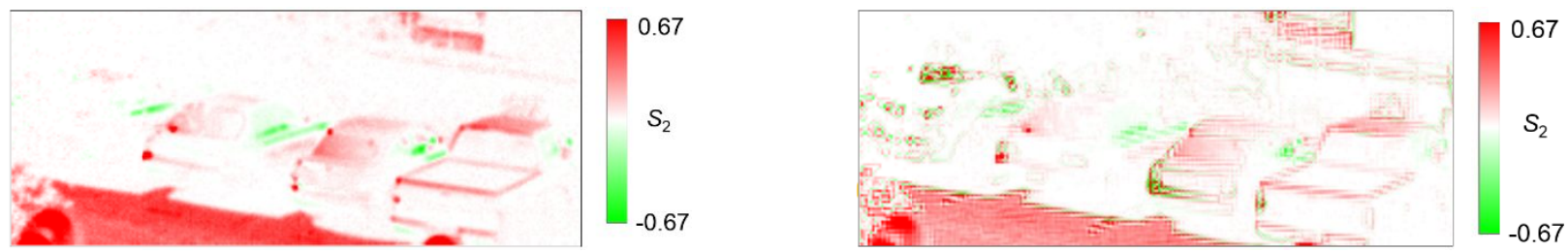

Figure 4. (Left) The measured MSPI $S_{2}$ image and (right) the $S_{2}$ image measured with a division of focal plane polarimeter. The difference between the two images characterizes the polarization artifacts for this image. Again the checkerboard structure is seen in the image on the right.

\section{CONCLUSION}

Many polarization images and spectra, even those published, are inaccurate. In our polarization laboratory where rigorous polarimeter operating procedures are in place, many data acquisition runs are evaluated then discarded as dubious and re-measured. There are problems with stray light, monochromator calibration and drift, ambient lights coming on in the room during measurements, misaligned samples, new software problems, and issues related to the building ventilation systems and power supply. It is recommended that all polarization measurements be approached with a degree of skepticism until the measurement system and measurement circumstances are clearly understood and appropriate tests and calibrations are provided.

\section{REFERENCES}

[1] i Russell A. Chipman, "Polarimeter calibration error gets far out of control," Proc. SPIE 9583, An Optical Believe It or Not: Key Lessons Learned IV, 95830H, 2015.

[2] Matt Novak, et al, "Analysis of a micropolarizer array-based simultaneous phase-shifting interferometer," Applied

Optics 44.32 (2005): 6861-6868. 
[3] $]^{\text {iii }}$ C. S. Chun, Fleming, D. L., Harvey, W. A., \& Torok, E. J. (1995, September). "Polarization-sensitive thermal imaging sensor," In SPIE's 1995 International Symposium on Optical Science, Engineering, and Instrumentation (pp. 438-444). International Society for Optics and Photonics.

$[4]^{\mathrm{iv}}$ G. P. Nordin, J. T. Meier, P. C. Deguzman, and M. W. Jones, "Micropolarizer array for infrared imaging polarimetry,” J. Opt. Soc. Am. A 16(5), 1168-1174 (1999).

[5] $]^{\mathrm{v}}$ Graham Myhre, Arshad Sayyad, and Stanley Pau, "Patterned color liquid crystal polymer polarizers," Optics express 18.26 (2010): 27777-27786.

$[6]^{\mathrm{vi}}$ Pochi Yeh, “A new optical model for wire grid polarizers,” Optics Communications 26.3 (1978): 289-292.

[7] ${ }^{\text {vii }}$ Stephen Arnold, et al. "An Improved Polarizing Beamsplitter LCOS Projection Display Based on Wire-Grid Polarizers," SID Symposium Digest of Technical Papers. Vol. 32. No. 1. Blackwell Publishing Ltd, 2001. 